\title{
Automatic Segmentation of Antenatal 3-D Ultrasound Images
}

\author{
Jérémie Anquez, Elsa D. Angelini, Senior Member, IEEE, Gilles Grangé, and Isabelle Bloch*, Member, IEEE
}

\begin{abstract}
The development of 3-D ultrasonic probes and 3-D ultrasound (3DUS) imaging offers new functionalities that call for specific image processing developments. In this paper, we propose an original method for the segmentation of the utero-fetal unit (UFU) from 3DUS volumes, acquired during the first trimester of gestation. UFU segmentation is required for a number of tasks, such as precise organ delineation, 3-D modeling, quantitative measurements, and evaluation of the clinical impact of 3-D imaging. The segmentation problem is formulated as the optimization of a partition of the image into two classes of tissues: the amniotic fluid and the fetal tissues. A Bayesian formulation of the partition problem integrates statistical models of the intensity distributions in each tissue class and regularity constraints on the contours. An energy functional is minimized using a level set implementation of a deformable model to identify the optimal partition. We propose to combine Rayleigh, Normal, Exponential, and Gamma distribution models to compute the region homogeneity constraints. We tested the segmentation method on a database of 19 antenatal 3DUS images. Promising results were obtained, showing the flexibility of the level set formulation and the interest of learning the most appropriate statistical models according to the idiosyncrasies of the data and the tissues. The segmentation method was shown to be robust to different types of initialization and to provide accurate results, with an average overlap measure of 0.89 when comparing with manual segmentations.
\end{abstract}

Index Terms - Antenatal imaging, biomedical image processing, image segmentation, level sets, 3-D ultrasonic imaging.

\section{INTRODUCTION AND LITERATURE}

$\mathbf{U}$ LTRASOUND imaging, introduced for obstetrical screening in the 1950s, became widely used as a diagnostic tool in the late 1960s, and was introduced as a screening tool for pregnancy monitoring in the late 1970s [1]. It remains the modality of choice for routine fetal imaging [2]. Obstetrical echography covers several applications including precise determination of the pregnancy stage, placenta positioning, fetal growth, or char-

Manuscript received April 23, 2012; revised August 25, 2012, November 9, 2012, and December 27, 2012; accepted December 27, 2012. Date of publication January 1, 2013; date of current version April 15, 2013. This work was supported in part by Orange Labs R\&D and Fondation Santé et Radiofréquences (FEMONUM Project). Asterisk indicates corresponding author.

J. Anquez is with Theraclion, 92240 Malakoff, France (e-mail: jeremie.anquez@gmail.com).

E. D. Angelini is with the Institut Mines-Telecom, Telecom ParisTech, CNRS LTCI, 75013 Paris, France (e-mail: elsa.angelini@ telecom-paristech.fr).

G. Grangé is with the Maternité Port Royal AP-HP, Groupe Hospitalier Cochin Saint Vincent De Paul, 75014 Paris, France (e-mail: gilles.grange@cch.aphp.fr).

${ }^{*}$ I. Bloch is with the Institut Mines-Telecom, Telecom ParisTech, CNRS LTCI, 75013 Paris, France (e-mail: isabelle.bloch@enst.fr).

Color versions of one or more of the figures in this paper are available online at http://ieeexplore.ieee.org.

Digital Object Identifier 10.1109/TBME.2012.2237400 acterization of potential pathologies [3]. Standard echography screening includes the acquisition of a series of 2-D B-scan anatomical images. These images are used for visual inspection or to perform quantitative measures of biometric markers such as biparietal diameter, femur length, head circumference, or abdominal circumference [4]. Three-dimensional echography was introduced in the early 1990s for fetal screening, but its widespread was limited due to poor image quality and slow acquisition protocols, unable to prevent fetal motion artifacts [5]. These limitations are progressively disappearing with advanced technologies, increasing the clinical interest for 3-D ultrasound (3DUS) [6]. During the first trimester and early stage of the second trimester of gestation, the field of view of the ultrasound probes can integrate the whole gestational sac. Consequently, 3DUS-based volumetric studies of uterine structures have been published [7], as well as quantification of the whole fetus [8] or partial body portions (e.g., head and trunk) [9], providing useful information for clinical routine. These volumetric studies still rely on manual tracing, and automated segmentation methods are, therefore, desirable. Semi-automated methods were used in recent studies, especially with the software tool VOCAL, commercialized by General Electric and cited in several works [7], [9], [10]. It enables to reconstruct smooth organ surfaces from a set of 2-D contours acquired on rotated views along a single axis [11]. This software remains limited to the extraction of single organs and is not yet capable of segmenting complex objects such as the whole fetus. Moreover, several manual interactions are often needed. Other works have dealt with the segmentation of specific organs, such as the cardiac cavities [12], using a commercial segmentation tool, and manually supervised image partition.

The general domain of automated ultrasound image segmentation was reviewed in [13] and includes dedicated methods for the extraction of biometric markers on fetal US imaging. A first family of 2-D methods proposes to segment specific anatomical structures with morphological operators, such as the femur in [14], and the skull in [15]. A second family of methods is based on deformable models. A parametric active contour [16], exploiting local intensity variations, was used in [17] to segment the skull. This approach was limited by the requirements to initialize the contour close to the skull borders and the lack of robustness on images with poor skull contrast. In [18], a Bayesian parametric deformable model was proposed, exploiting statistical models of intensity distributions in the femoral and skull bones. Recent works in [19] have enabled the segmentation of the skull, abdomen, and femoral bone.

Few works were dedicated to automated segmentation of 3DUS images for obstetrical applications, although there is 
a real clinical need for automated and reproducible methods leading to reliable quantitative measurements. We can cite the preliminary work of Sarti et al. [20] where the so-called subjective surfaces were proposed to segment objects with partially missing contours. In [21], these surfaces were improved with additional homogeneity terms, to segment fetal heart cavities on two datasets. More recently, the method proposed in [22] uses a maximum likelihood (ML) formalism optimized with level set deformable models. Intensities inside and outside the fetus were modeled with Rayleigh distributions, but no clinical validation of the method was reported. In [23], the authors derived a multiple object detection framework evaluated on 3DUS fetal ultrasound for brain structures and faces. Binary classifiers, indicating the presence or absence of an object, were learned from a large database of annotated images. In [24], the authors proposed a method for fetal face extraction from 3DUS data. Multiscale Haar expansions and steerable filters were used to derive image features on face landmark points. The same features were used in [25] for the supervised learning and detection of fetal anatomical structures.

In this paper, we address the problem of fetal 3DUS volume segmentation as an optimal partition of the voxels into statistically "homogeneous" regions (corresponding to different classes) with smooth contours. We use parametric probability distribution functions (pdf) to measure region homogeneities, as in [22]. The classification task is formulated as a variational deformable model segmentation, within a level set framework, and performed through an iterative deformation process of an initial shape. As an original feature of the method, we propose to rely on a generic class of pdf which can model different types of tissue distributions in both saturated and non-saturated images. This differs from existing methods such as [22]-[25].

Segmentation results were evaluated quantitatively with the help of an experienced obstetrician, who provided some manual segmentations. Experiments on 19 obstetrical ultrasound 3-D images illustrate the behavior of the method and the influence of the parameters. Quantitative results show accurate and robust segmentation performances.

\section{3DUS IMAGES AND TISSUE APPEARANCE MODELS}

\section{A. Image Database}

A database of 3DUS volumes (noted $D B=\left\{I_{i}\right\}_{i=1 \ldots 19}$ ) was gathered, including:

1) eighteen $3-\mathrm{D}$ volumes $\left\{I_{i}\right\}_{i=1 \ldots 18}$ provided by the Beaujon AP-HP hospital (Clichy, France) and acquired with a Voluson 730 Expert system from General Electric (GE, Zipf, Austria), with a 3.7-9.3 MHz transvaginal volumetric probe. Spatial resolution ranges from 0.21 to $0.96 \mathrm{~mm}^{3}$ with isotropic voxels;

2) and one $3-\mathrm{D}$ volume $I_{19}$ provided by Philips Healthcare Research Labs (Suresnes, France) and acquired with a iU22 transducer from Philips Ultrasound (Bothell, WA, USA), with a 2-6 MHz volumetric probe. Spatial resolution was $0.95 \times 0.6 \times 1.37 \mathrm{~mm}^{3}$.

This database enabled us to study a large set of examples of fetuses in different positions and at different stages of ges- tation during the first trimester of pregnancy. All cases in the database were associated with their timing of acquisition, measured as the number of weeks of amenorrhea (WA) of the mother, which ranged between 8 and 13 WA for $\left\{I_{i}\right\}_{i=1 \ldots 18}$. The additional case, $I_{19}$, acquired at $22 \mathrm{WA}$, was also included in the database. Voluson images were acquired using harmonic imaging and without compounding. This corresponds to the routine acquisition mode used at the collaborating hospital for obstetric 3DUS acquisitions during the first trimester, to optimize image contrast at the interfaces between tissues. The echographers defined a field of view as small as possible, while including the whole amniotic sac. In some cases, the amniotic sac was, however, slightly truncated. Images were post-processed (for speckle reduction) and reconstructed on a Cartesian grid with dedicated post-processing tools provided by the manufacturers and included in the ultrasound scanning systems. Voxel intensities were normalized during this process in the range of values $[0,255]$. These considerations have an impact on the modeling of the intensity distributions, as explained later.

Given the low signal-to-noise ratio (SNR) of ultrasound image data, setting up an automated segmentation of homogeneous structures is a difficult task. Indeed, images are corrupted with textured speckle noise and some structures lack sharp contrast along their contours. Visual inspection of the data by an obstetrician relies heavily on prior knowledge of the fetal anatomy, and the specific image characteristics generated by the presence of highly reflective interfaces, absorption in bone structures, and echo cancellation along interfaces parallel to the ultrasound beam. To learn tissue appearance and validate the segmentation method presented in this paper, an experienced obstetrician interactively processed the images with two different approaches: 1) detailed manual segmentation of some fetal and maternal structures, 2) binarization of the images into amniotic fluid and fetal tissues via intensity thresholding. These two approaches are now detailed.

1) Manual segmentation of 3DUS volumes is a tedious task given their large size and their low SNR. Therefore, only a subset of five volumes was manually segmented in 3-D. The set of volumes included four development stages of the fetuses over the first trimester, corresponding to 8-9-10-13 WA, and the case at 22 WA. The placenta, amniotic fluid, fetus envelope, and umbilical cord were manually delineated by an experienced obstetrician. Slices from the 9 and 13 WA cases are illustrated in Fig. 1(a) and (b), with the corresponding detailed manual segmentations. For the other volumes of the database, three slices in orthogonal directions were segmented to outline the spatial extent of the anatomical structures of interest. Two voxel classes were distinguished: the amniotic fluid and the fetal tissues (including the placenta, ${ }^{1}$ umbilical cord, and fetus), as illustrated in Fig. 1(c) and (d). The two classes, included in these partial segmentations, are denoted AF and FT, respectively.

2) Using an interactive software tool, the experienced obstetrician interactively defined for each volume case-specific threshold values to binarize the volumes into two regions, corresponding to $\mathrm{AF}$ and $\mathrm{FT}$.

\footnotetext{
${ }^{1}$ The placenta is indeed a fetal tissue originating from the feconded egg.
} 


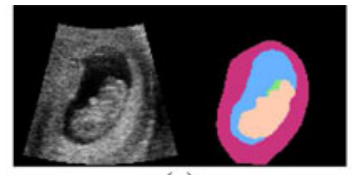

(a)

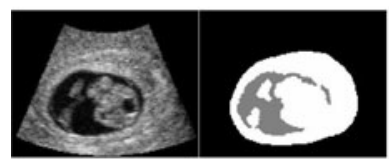

(c)

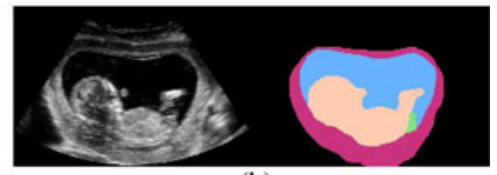

(b)

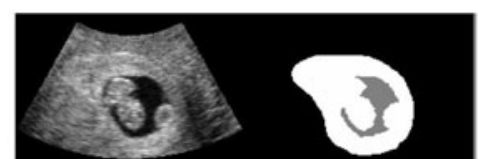

(d)
Fig. 1. (a) and (b) Two-dimensional slices of $I_{9}$ (9 WA), $I_{18}$ (13 WA) with the corresponding complete manual segmentations. Segmented tissues are the placenta (red), the amniotic fluid (blue), the fetus (pink), and the umbilical cord (green). (c) and (d) Two orthogonal slices of $I_{6}$ (9 WA) with the corresponding partial manual segmentations. Identified tissues are the amniotic fluid (gray) and fetal tissues (white)

Our segmentation framework is designed to be used with any type of initialization (e.g., random-uniform partitions or thresholding with an a priori threshold). We used the manually defined thresholds to learn an average threshold value, that can be used for the initial partition of any new volume of images to segment, prior to fine level-set segmentation. This initialization greatly speeds up the convergence of the segmentation algorithm. Two criteria were considered to define the manual threshold values: 1) the completeness of the boundary between the placenta and the amniotic fluid, and 2) the precision of the position of the boundary between the fetus and the amniotic fluid. A first set of threshold values $\left\{s_{i}^{1}\right\}$ was defined for each volume of images $I_{i}$ during a first session. One week later, a second session was held to identify a second set of values $\left\{s_{i}^{2}\right\}$. These consecutive sessions enabled us to assess the intra-expert variability in defining a single optimal threshold value per case. The mean value $s_{i}$ between the two experiments was computed. Let $\epsilon_{i}=\left|s_{i}^{1}-s_{i}^{2}\right|$ be the absolute difference between the two thresholds for each $I_{i}$. Let $\mu_{\epsilon}$ and $\sigma_{\epsilon}$ be the mean and standard deviation of the $\epsilon_{i}$ values, respectively. A noticeable intra-user variability was measured, since $\mu_{\epsilon}=12.8$ and $\sigma_{\epsilon}=5.6$, which can be explained by the important amount of noise in the images. The quantity $\epsilon^{+}=\max _{i}\left(\epsilon_{i}\right)$ can be used to define an interval of "admissible" threshold values for each volume of the database: a value $s$ is considered admissible to binarize $I_{i}$ if it satisfies $s \in\left[s_{i}^{-}, s_{i}^{+}\right]$, with $s_{i}^{+}=s_{i}+\frac{\epsilon^{+}}{2}$ and $s_{i}^{-}=s_{i}-\frac{\epsilon^{+}}{2}$. Let $\mu_{i}^{s}$ be the average of the mean threshold values $s_{i}$ over the whole database but excluding the volume $I_{i}$. These average values $\mu_{i}^{s}$ ended up to be admissible threshold values for 17 of the 19 volumes of images of the database. For the other two volumes, the average still remained close to the $s_{i}$ values (less than five gray levels difference).

Alternative threshold values were also studied, using the Otsu's automatic thresholding method [26] and the K-means classification [27] with $K=2$. The same threshold values were obtained with the two automated selection methods. Let $o_{i}$ be the threshold value for $I_{i}$. The $o_{i}$ threshold values were higher than the $s_{i}$ values and were non-admissible (in a strict sense) in 17 of the 19 cases. However, the distances $\left|o_{i}-s_{i}\right|$ remained small compared to the intensity range in the images (the average $\left|o_{i}-s_{i}\right|$ distance was inferior to 25 , which is equivalent to $10 \%$ of the whole intensity range).

\section{B. Intensity Distribution Modeling}

Distributions of voxel intensities were learned based on the manual segmentation presented above. Two sets of voxels were considered: $\Omega_{\mathrm{AF}}$, for the voxels belonging to $\mathrm{AF}$, and $\Omega_{\mathrm{FT}}$, for the voxels belonging to FT. During the ultrasound data acquisition process, a transfer function can be applied to saturate the low and high intensities, reducing the range of attenuations to record and facilitating image interpretation by the clinician, within soft tissues. As a result, some images of the database were saturated by the ultrasound scanning machine during the acquisition. We therefore distinguished two subsets within the database: $D B_{S}$ which contains saturated volumes and $D B_{\bar{S}}$ which contains the non-saturated volumes. This was simply done by identifying the presence of an initial large peak in the histogram for saturated cases. In this study, histograms were reviewed manually to detect the presence of this peak and label each case in the database as having saturated or unsaturated intensities in the amniotic fluid. We identified $D B_{\bar{S}}=\left\{I_{i}\right\}, i \in\{1, \ldots, 5\}$ and $D B_{S}=\left\{I_{i}\right\}, i \in\{6, \ldots, 19\}$. Histograms of the intensity values within $\Omega_{\mathrm{AF}}$ and $\Omega_{\mathrm{FT}}$ are noted $\hat{p}_{i}^{\mathrm{AF}}$ and $\hat{p}_{i}^{\mathrm{FT}}$ for the volume $I_{i}$, and can differ significantly.

Standard modeling of tissue intensity distributions in US images has been based on the Rayleigh distribution in many works. However, it did not provide satisfying modeling on our database and may not properly model tissue intensities on clinical ultrasound images as discussed below. Alternatively, we propose to exploit the Gamma distribution, which pdf is expressed as

$$
p_{G}(I(x))=I(x)^{\alpha-1} \frac{\beta^{\alpha} \exp (-\beta I(x))}{\Gamma(\alpha)}
$$

with $I(x)$ the image intensity at voxel $x$ (with $I(x)>0$ ), $\alpha>$ $0, \beta>0$, and

$$
\Gamma(\alpha)=\int_{0}^{\infty} t^{\alpha-1} e^{-t} d t
$$

This pdf provides great genericity and flexibility for the modeling of voxel intensities, through the parameters $\alpha$ and $\beta$. The ML estimators $\alpha_{\mathrm{ML}}$ and $\beta_{\mathrm{ML}}$ of the distribution parameters $\alpha$ and $\beta$ were evaluated for each dataset. Since no close form exists for $\alpha_{\mathrm{ML}}$, this value was estimated using the method presented in [28], based on the iterative Newton's technique. The value of $\beta_{\mathrm{ML}}$ was then computed, which depends only on $\alpha_{\mathrm{ML}}$.

Modeling of the intensity distributions using the Gamma pdf is illustrated in Fig. 2. The histograms $\hat{p}_{4}^{\mathrm{AF}}$ (a), $\hat{p}_{4}^{\mathrm{FT}}$ (b), $\hat{p}_{13}^{\mathrm{AF}}$ (c), and $\hat{p}_{13}^{\mathrm{FT}}$ (d) are displayed, along with the corresponding pdf $p_{G}(I(x))\left(I_{4}\right.$ corresponds to a non-saturated volume, while $I_{13}$ is a saturated one). The fitting ability of the distribution is not surprising in the non-saturated case. It was also used advantageously in [29] to model blood appearance (a physiological fluid with a similar appearance to the amniotic fluid) and soft tissue appearance on echocardiographic images. More interestingly, its genericity also enables to model the intensities when saturated images are considered as illustrated in Fig. 2(c) 


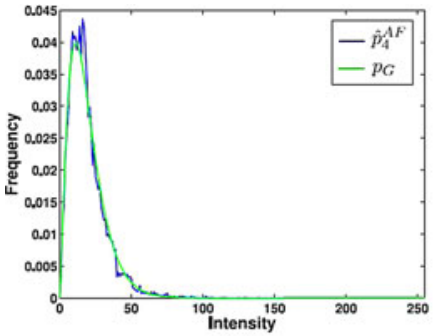

(a)

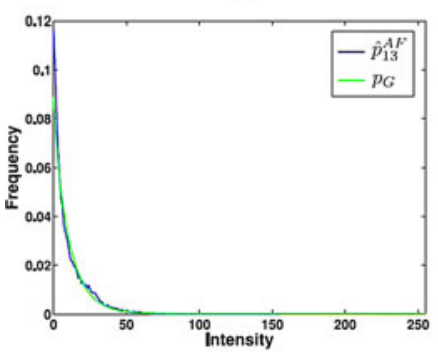

(c)

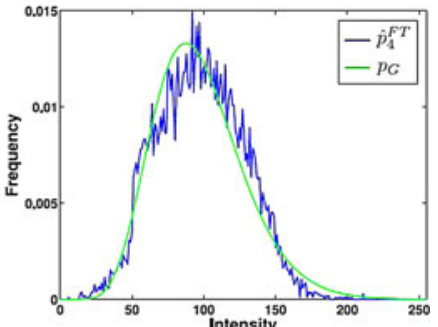

(b)

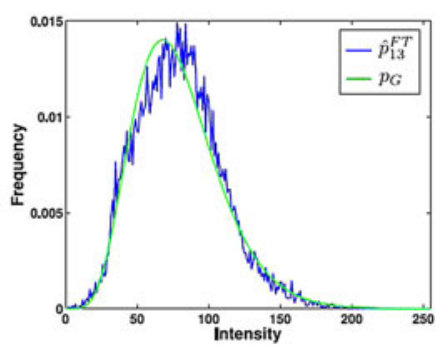

(d)

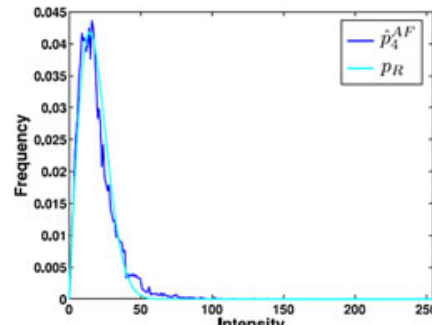

(a)

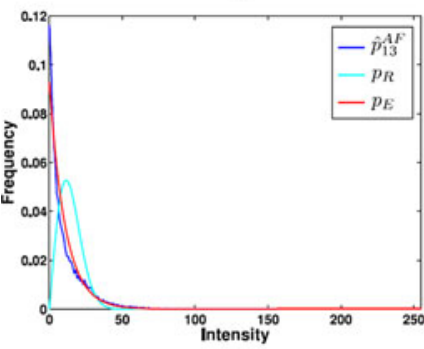

(c)

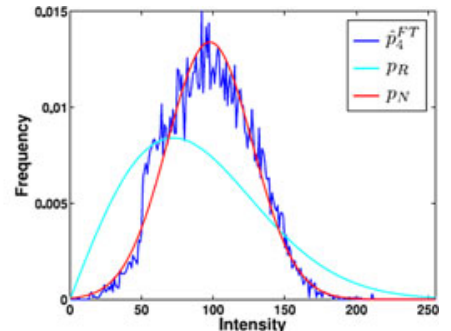

(b)

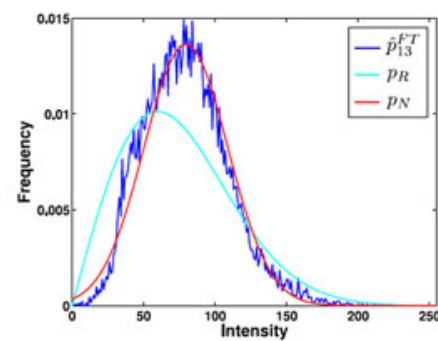

(d)

Fig. 2. Modeling of the intensity distributions with the Gamma pdf in $\Omega_{\mathrm{AF}}$ and $\Omega_{\mathrm{FT}}$ : (a), (b) in the non-saturated volume $I_{4}$ and (c), (d) in the saturated volume $I_{13}$. The histograms: (a) $\hat{p}_{4}^{\mathrm{AF}}$, (b) $\hat{p}_{4}^{\mathrm{FT}}$, (c) $\hat{p}_{13}^{\mathrm{AF}}$, and (d) $\hat{p}_{13}^{\mathrm{FT}}$ are represented in blue and the Gamma distribution $p_{G}(I(x))$ in green.

and (d). This shows that the Gamma distribution can be used as a generic intensity model for the AF and the FT, whatever post-processing was applied to the US signal when recorded by the scanning system, but at the cost of computing distribution parameters that do not have analytical expressions.

As an alternative, we investigated the use of tissue-specific distribution models, with analytical ML estimators of the parameters. Depending on the intensity saturation, we propose to use different parametric distributions, dedicated to the two specific tissue types being studied (amniotic fluid or fetal tissues). Regarding the amniotic fluid, we exploit the Rayleigh distribution to model the intensity distribution in non-saturated images. This distribution, initially proposed in [30] and [31], has been widely used for ultrasound images processing [13]. The pdf of this distribution is expressed as:

$$
p_{R}(I(x))=\frac{I(x) \exp \left(\frac{-I(x)^{2}}{2 \sigma^{2}}\right)}{\sigma^{2}}
$$

with $I(x) \in[0, \infty)$ and $\sigma>0$. This distribution provides a good fit of the intensity distribution in the amniotic fluid of a nonsaturated image, as illustrated in Fig. 3(a). However, this is no longer true when the intensities are saturated. In this case, the frequency of the zero intensity class is artificially high. Consequently, the Rayleigh distribution is not relevant since it always leads to $p_{R}(0)=0$. Moreover, the histogram value decreases, while $p_{R}(I(x))$ increases for $I(x) \in[0, \sigma]$ and decreases for $I(x) \in[\sigma, \infty)$. As a surrogate, we exploit the Exponential distribution to model the intensity histograms in $\Omega_{\mathrm{AF}}$, when $I_{i} \in D B_{S}$. The pdf of this distribution is expressed as:

$$
p_{E}(I(x))=\lambda e^{-\lambda I(x)}
$$

with $I(x) \in[0, \infty)$ and $\lambda>0$. This choice clearly fits better the intensity histograms than the Rayleigh distribution [see

Fig. 3. Modeling of the intensity distributions with specific models in $\Omega_{\mathrm{AF}}$ and $\Omega_{\mathrm{FT}}$ (a), (b) in the non-saturated image $I_{4}$ and (c), (d) in the saturated image $I_{13}$. The histograms (a) $\hat{p}_{4}^{\mathrm{AF}}$, (b) $\hat{p}_{4}^{\mathrm{FT}}$, (c) $\hat{p}_{13}^{\mathrm{AF}}$, and (d) $\hat{p}_{13}^{\mathrm{FT}}$ are represented in blue and the Rayleigh distribution $p_{R}(I(x))$ in cyan (a)-(d). (b), (d) Normal and (c) Exponential distributions are represented in red.

Fig. 3(c)]. This distribution has been seldom considered to model intensity distributions in ultrasound images, since saturated images are frequently excluded from the test databases used to validate image processing methods [29]. It has nevertheless been exploited to model the blood and to segment echocardiographic images [32]. Regarding fetal tissues, one can notice by observing $\hat{p}_{4}^{\mathrm{FT}}$ and $\hat{p}_{13}^{\mathrm{FT}}$ in Fig. 3(b) and (d) that there are very few pixels with intensity below 25 , which actually belong to regions affected by acoustic shadowing. Generally, the $\hat{p}_{i}^{\mathrm{FT}}$ increase rate is slow for low intensities. The Rayleigh distribution is therefore not satisfying to model the intensity distributions in $\Omega_{\mathrm{FT}}$, and we propose to use the Normal distribution. The pdf of this distribution is expressed as

$$
p_{N}(I(x))=\frac{1}{\sigma \sqrt{2 \pi}} \exp \left(-\frac{(I(x)-\mu)^{2}}{2 \sigma^{2}}\right)
$$

with $I(x) \in \mathbb{R}, \mu \in \mathbb{R}, \sigma>0$. It was also used to model the intensity distribution in soft tissues, to process ultrasound images of the heart and prostate in [33] and [34].

An important advantage of the Rayleigh, Exponential, and Normal distributions over the Gamma distribution is that close forms of the ML estimators of their parameters systematically exist. This simplifies the fitting process, in comparison with the computation of the Gamma distribution parameter $\alpha_{\mathrm{ML}}$, which is obtained through an iterative process. Choice is left to the user to give priority either to the genericity of the model or to the computation speed.

To quantitatively evaluate the goodness of fit between the histograms and our models, we used the Cramer-Von Mises criterion [35] on the whole database $D B$ and for the two classes of tissues. This criterion, $C V M$, is expressed as $C V M=$ $\int_{-\infty}^{+\infty}\left(P^{*}(g)-P(g)\right)^{2} d P(g)$, comparing the cumulative 
TABLE I

DESCRIPTION OF THE INTENSITY DISTRIBUTION MODELING STRATEGIES USED TO GUIDE THE SEGMENTATION OF THE 3DUS VOLUMES

\begin{tabular}{|c|c|c|}
\hline Modeling strategy & Distributions $\left(\Omega_{A F} / \Omega_{F T}\right)$ & Notation \\
\hline Generic distributions & Gamma/Gamma & GG \\
\hline Specific distributions in $D B_{\bar{S}}$ & Rayleigh/Normal & SD (RN) \\
\hline Specific distributions in $D B_{S}$ & Exponential/Normal & SD (EN) \\
\hline Chan and Vese [36] & $\begin{array}{c}\text { Normal/Normal } \\
\text { (with identical st. dev.) }\end{array}$ & CV \\
\hline
\end{tabular}

density function of the theoretical distribution $P$ with the cumulative histogram of the empirical data $P^{*}$. This test has the advantage of being weakly sensitive to the tail of the distributions. Since this tail may not be well represented in the experimental data, and thus wrongly estimated, it is better to use a test that is not too sensitive to it. The CVM test was therefore preferred to the Kolomogorov-Smirnov one, which is more sensitive to outliers and wrong estimation of the tail of the distribution. The lower the CVM test value, the better the fit between the data and the distribution model. We obtained optimal test values with the Gamma model, followed by the Exponential (for AF)/Normal (for FT) model and the Rayleigh model. Average $C V M$ test values for $\{\mathrm{AF}, \mathrm{FT}\}$ tissues with the three models were: $\{0.001,0.001\}$ for the Gamma model, $\{0.01,0.001\}$ for the Exponential/Normal model and $\{0.037,0.0075\}$ for the Rayleigh model. For the Rayleigh model, we observed a large variability of the $C V M$ values over the 19 cases, and the maximum values were 0.08 and 0.02 for $\mathrm{AF}$ and FT tissues.

This statistical evaluation confirmed that the Gamma distribution provides a relevant intensity modeling in $\Omega_{\mathrm{AF}}$ and $\Omega_{\mathrm{FT}}$, for saturated and unsaturated images. Fitting accuracy was equivalent or better than with specific distributions in $\Omega_{\mathrm{AF}}$, while being slightly inferior than with the Normal distribution for 15 cases of the $D B$ in $\Omega_{\mathrm{FT}}$.

The segmentation process presented in the next section relies on intensity distributions modeling in $\Omega_{\mathrm{AF}}$ and $\Omega_{\mathrm{FT}}$, which requires iterative data fitting and parameters estimation. The results obtained with the dedicated models will be compared with a strategy consisting in modeling voxels intensities in a given region using Normal distributions with identical standard deviations. This model corresponds to the Chan-Vese method described in [36].

The intensity distribution modeling strategies used to guide the segmentation process are summarized in Table I. Note that if using the SD modeling strategy (with distributions depending on the tissues), information on the presence of saturation or not is used to decide on using an Exponential or a Rayleigh distribution to model the amniotic fluid.

\section{Segmentation Method: Bayesian Formulation AND VARIATIONAL APPROACH}

Let $\Omega$ be an open and bounded subset of $\mathbb{R}^{N}$ and let $I: \Omega \rightarrow \mathbb{R}$ be an N-D image to segment. We propose a method which aims at providing an optimal partition of the image domain, noted $\mathcal{P}(\Omega)=\left\{\Omega_{e}, \Omega_{i}\right\}$, by embedding prior information regarding the distributions followed by the voxel intensities in $\Omega_{e}$ and $\Omega_{i}$ and on the boundary between these two regions $\left(\Omega_{i}\right.$ and $\Omega_{e}$ correspond to $\Omega_{\mathrm{AF}}$ and $\Omega_{\mathrm{FT}}$ ). To achieve this task, we consider a Bayesian framework and propose to maximize the posterior probability of the partition given the image $I$, denoted $p(\mathcal{P}(\Omega) \mid I)$. Since $p(I)$ is identical for all partitions, this is equivalent to maximizing $p(I \mid \mathcal{P}(\Omega)) p(\mathcal{P}(\Omega))$. The first term is called the image likelihood and the second term represents the a priori probability of the partition and is modeled as a boundary smoothness constraint. Maximization of $p(\mathcal{P}(\Omega) \mid I)$ corresponds to the identification of the maximum a posteriori (MAP) partition $\mathcal{P}(\Omega)$.

\section{A. Formulation of the Boundary Smoothness Constraint}

Let $C$ be the boundary of the partition $\mathcal{P}(\Omega)$. Integrating a prior on the boundary enables to regularize the optimization problem. This is particularly important in the case of US images, as underlined, for example, in [37]. We aim at modeling smooth boundaries, since this feature characterizes most organs and anatomical structures. To obtain smooth boundaries, we consider the following prior that depends on the measure $|C|$ of the boundary $C$ (curve in 2-D, surface in 3-D) between $\Omega_{e}$ and $\Omega_{i}$ (i.e. the length in 2-D and the surface area in 3-D):

$$
p(\mathcal{P}(\Omega)) \propto \nu \exp (-\nu|C|), \nu>0 .
$$

The smoothness of the boundary $C$ is controlled by the parameter $\nu$ of this distribution. The choice of $\nu$ is explained in Section IV-A.

\section{B. Formulation of the Region Likelihood}

The likelihood term relies on a homogeneity measure computed at each voxel $x$ with intensity $I(x)$ in the image, which depends on the region it belongs to. The pdf $p(I(x))$ then takes a first form $p_{e}$ with parameter(s) $\theta_{e}$ in a parameter space $\Theta_{e}$, hence denoted $p_{e}\left(I(x), \theta_{e}\right)$, if $x \in \Omega_{e}$, and a second form $p_{i}$ with parameter(s) $\theta_{i}$ in a parameter space $\Theta_{i}$ (denoted $p_{i}\left(I(x), \theta_{i}\right)$ ), if $x \in \Omega_{i}$. Consequently, we have

$$
p(I(x))= \begin{cases}p_{e}\left(I(x), \theta_{e}\right), & \text { if } x \in \Omega_{e}, \text { with } \theta_{e} \in \Theta_{e} \\ p_{i}\left(I(x), \theta_{i}\right), & \text { if } x \in \Omega_{i}, \text { with } \theta_{i} \in \Theta_{i} .\end{cases}
$$

Under the hypothesis that the voxel intensities are independent conditionally to $\mathcal{P}(\Omega)$, we obtain

$$
p(I \mid \mathcal{P}(\Omega))=\prod_{x \in \Omega_{e}} p_{e}\left(I(x), \theta_{e}\right) \prod_{x \in \Omega_{i}} p_{i}\left(I(x), \theta_{i}\right) .
$$

\section{Posterior Probability}

Integrating (6) and (8) into the posterior probability of a partition $\mathcal{P}(\Omega)$ conditionally to the image leads to:

$p(\mathcal{P}(\Omega) \mid I)=\nu \exp (-\nu|C|) \prod_{x \in \Omega_{e}} p_{e}\left(I(x), \theta_{e}\right) \prod_{x \in \Omega_{i}} p_{i}\left(I(x), \theta_{i}\right)$.

\section{Formulation of the MAP Optimization Problem}

To solve the MAP problem, we linearize the posterior probability by defining an energy $E$ equal to the negative logarithm of $p(\mathcal{P}(\Omega) \mid I)$. The negative logarithm function being 
strictly decreasing, the minimization of $E$ is equivalent to the maximization of $p(\mathcal{P}(\Omega) \mid I)$. Therefore, we look for $C, \theta_{e}, \theta_{i}$ minimizing $E$ :

$$
E\left(C, \theta_{e}, \theta_{i}\right)=E_{\mathrm{reg}}(C)+E_{e}\left(C, \theta_{e}\right)+E_{i}\left(C, \theta_{i}\right)
$$

with

$$
\left\{\begin{array}{l}
E_{\mathrm{reg}}(C)=-\log \nu+\nu|C| \\
E_{e}\left(C, \theta_{e}\right)=-\int_{x \in \Omega_{e}} \log p_{e}\left(I(x), \theta_{e}\right) d x \\
E_{i}\left(C, \theta_{i}\right)=-\int_{x \in \Omega_{i}} \log p_{i}\left(I(x), \theta_{i}\right) d x .
\end{array}\right.
$$

Note that the integrals become discrete sums over a bounded domain in the implementation on digital images. The energy $E$ is minimized by optimizing its three parameters $\left(C, \theta_{e}, \theta_{i}\right)$, noting that $\left\{\Omega_{e}, \Omega_{i}\right\}$ are entirely defined by the position of the contour $C$. The constant $-\log \nu$ of $E_{\text {reg }}$ is neglected in the following, since it depends on a weighting parameter $\nu$ that is not optimized during the minimization process of $E$, but fixed beforehand.

\section{E. Segmentation Via Energy Minimization}

Minimization of the energy functional $E$ is performed by an iterative process, progressively deforming an initial contour $C_{0}$ and updating the pdf parameters $\left\{\theta_{e}, \theta_{i}\right\}$. At each iteration, the contour is deformed to correspond to a lower energy level. To implement this iterative process, we need to encode the spatial localization of the contour $C$. To do this, we chose to use the level set framework which represents the contours $C$ implicitly as the zero level of a scalar function $\phi: \Omega \rightarrow \mathbb{R}$. The sign of $\phi$ therefore defines two regions: $\Omega_{e}$, where $\phi(x)>0$, and $\Omega_{i}$, where $\phi(x)<0$ [38]. The boundary $C$ between $\Omega_{e}$ and $\Omega_{i}$ is implicitly defined as $\phi(x)=0$.

To reformulate the energy functional in terms of $\phi$ instead of $\left\{\Omega_{e}, \Omega_{i}\right\}$ (or $C$ ), we express the measure $|C|$ in $E_{\text {reg }}(\phi)$ with the Dirac function $\delta(\phi)$, equal to zero everywhere except where $\phi(x)=0$. The terms $E_{e}$ and $E_{i}$ can be rewritten as integrals over the entire image, by exploiting the Heaviside function $H(\phi)$, equal to 1 where $\phi(x)>0$ and zero elsewhere. Equation (10) is, therefore, rewritten as:

$$
E\left(\phi, \theta_{i}, \theta_{e}\right)=E_{\mathrm{reg}}(\phi)+E_{e}\left(\phi, \theta_{e}\right)+E_{i}\left(\phi, \theta_{i}\right)
$$

with

$$
\left\{\begin{array}{l}
E_{\mathrm{reg}}(\phi)=\nu \int_{x \in \Omega} \delta(\phi(x))|\nabla \phi(x)| d x \\
E_{e}\left(\phi, \theta_{e}\right)=-\int_{x \in \Omega} H(\phi(x)) \log \left(p_{e}\left(I(x), \theta_{e}\right)\right) d x \\
E_{i}\left(\phi, \theta_{i}\right)=-\int_{x \in \Omega}(1-H(\phi(x))) \log \left(p_{i}\left(I(x), \theta_{i}\right)\right) d x .
\end{array}\right.
$$

The energy $E$ now depends on the three parameters $\left(\phi, \theta_{e}, \theta_{i}\right)$. The minimization of $E$ is performed using the numerical implementation detailed in [36]. This implementation alternates between two minimization tasks:

1) minimizing $E$ with respect to $\theta_{e}$ and $\theta_{i}$ (with a fixed $\phi$ ), and

2) minimizing $E$ with respect to $\phi$ (with fixed $\theta_{e}$ and $\theta_{i}$ parameters).

The energy $E$ being non-convex, only a local minimum might be obtained [39]. The quality of the initialization might,
TABLE II

ML Estimators $\hat{\theta_{r}}$ OF THE PARAMETER VALUES FOR THE PDF LAWS CONSIDERED IN THE HOMOGENEITY MEASURES $[r=e, i]$

\begin{tabular}{|c|c|}
\hline pdf law & $\hat{\theta_{r}}$ \\
\hline Normal & $\mu_{r}=\frac{1}{\left|\Omega_{r}\right|} \int_{x \in \Omega_{r}} I(x) \mathrm{d} x$ \\
& $\sigma_{r}=\left(\frac{1}{\left|\Omega_{r}\right|} \int_{x \in \Omega_{r}}\left(I(x)-\mu_{r}\right)^{2} \mathrm{~d} x\right)^{\frac{1}{2}}$ \\
\hline Rayleigh & $\sigma_{r}=\left(\frac{1}{2\left|\Omega_{r}\right|} \int_{x \in \Omega_{r}} I(x)^{2} \mathrm{~d} x\right)^{\frac{1}{2}}$ \\
\hline Exponential & $\lambda_{r}=\frac{\left|\Omega_{r}\right|}{\int_{x \in \Omega_{r}} I(x) \mathrm{d} x}$ \\
\hline Gamma & $\alpha_{r}=$ numerical approximation \\
& $\beta_{r}=\frac{1}{\alpha_{r}\left|\Omega_{r}\right|} \int_{x \in \Omega_{r}} I(x) \mathrm{d} x$ \\
\hline
\end{tabular}

therefore, play an important role, which is further discussed in Section IV-D.

Starting from an initial function $\phi_{0}(x)$ at time $t=0$, we define $\phi(x, 0)=\phi_{0}(x)$. This initial function is defined as the signed distance to the initial contour $C_{0}$. A gradient descent is then used to derive the following system governing the dynamic deformation of the implicit level set function, now noted $\phi(x, t)$ :

$$
\left\{\begin{array}{l}
\hat{\theta_{r}}=\underset{\theta_{r} \in \Theta_{r}}{\arg \min }\left(E_{r}\left(C, \theta_{r}\right)\right) \\
=\underset{\theta_{r} \in \Theta_{r}}{\arg \min }\left(-\int_{x \in \Omega_{r}} \log \left(p_{r}\left(I(x), \theta_{r}\right)\right) d x\right),[r=i, e] \\
\frac{\partial \phi}{\partial t}=\delta(\phi(x))\left(F_{\mathrm{reg}}(\phi(x))+F_{\text {data }}\left(\phi(x), \hat{\theta_{e}}, \hat{\theta_{i}}\right)\right) \text { in } \Omega
\end{array}\right.
$$

with

$$
\left\{\begin{array}{l}
F_{\text {reg }}(\phi(x))=\nu \operatorname{div}\left(\frac{\nabla \phi(x)}{|\nabla \phi(x)|}\right) \\
F_{\text {data }}\left(\phi(x), \hat{\theta_{e}}, \hat{\theta_{i}}\right)=F_{i}\left(\phi(x), \hat{\theta_{i}}\right)-F_{e}\left(\phi(x), \hat{\theta_{e}}\right) \\
F_{r}\left(\phi(x), \hat{\theta_{r}}\right)=-\log p_{r}\left(I(x), \hat{\theta_{r}}\right),[r=i, e]
\end{array}\right.
$$

where $F_{\mathrm{reg}}, F_{e}, F_{i}$ are derived from $E_{\mathrm{reg}}, E_{e}, E_{i}$, respectively, by calculating the partial derivatives with respect to $\phi$.

The expression of $\hat{\theta_{r}}$ in (11) corresponds to the definition of the ML estimate of the pdf parameter within each region $\Omega_{r}$. It is therefore explicitly defined according to the chosen distribution and the models listed in Table I. Closed-form expressions of the ML parameters exist for the Rayleigh, Exponential, and Normal distributions. Their derivation is described in [22] and [40]. For the Gamma distribution, a closed form of the scale parameter $\beta$ exists, but not for the shape parameter $\alpha$. This value is approximated with the method proposed in [28]. Expressions of the different $\theta_{r}$ are detailed in Table II for the different pdf laws considered in this study to measure homogeneity of appearance within 3DUS image regions.

\section{RESULTS}

In this section, we first discuss the implementation and parameterization of the segmentation method and then present an evaluation of the accuracy of the segmentation results on the database of obstetrical 3DUS volumes, comparing to manual tracing.

\section{A. Numerical Implementation of the Segmentation Method}

The pseudocode of the algorithm used to solve the system of equations (11) is described in Fig. 4, where we introduce some 


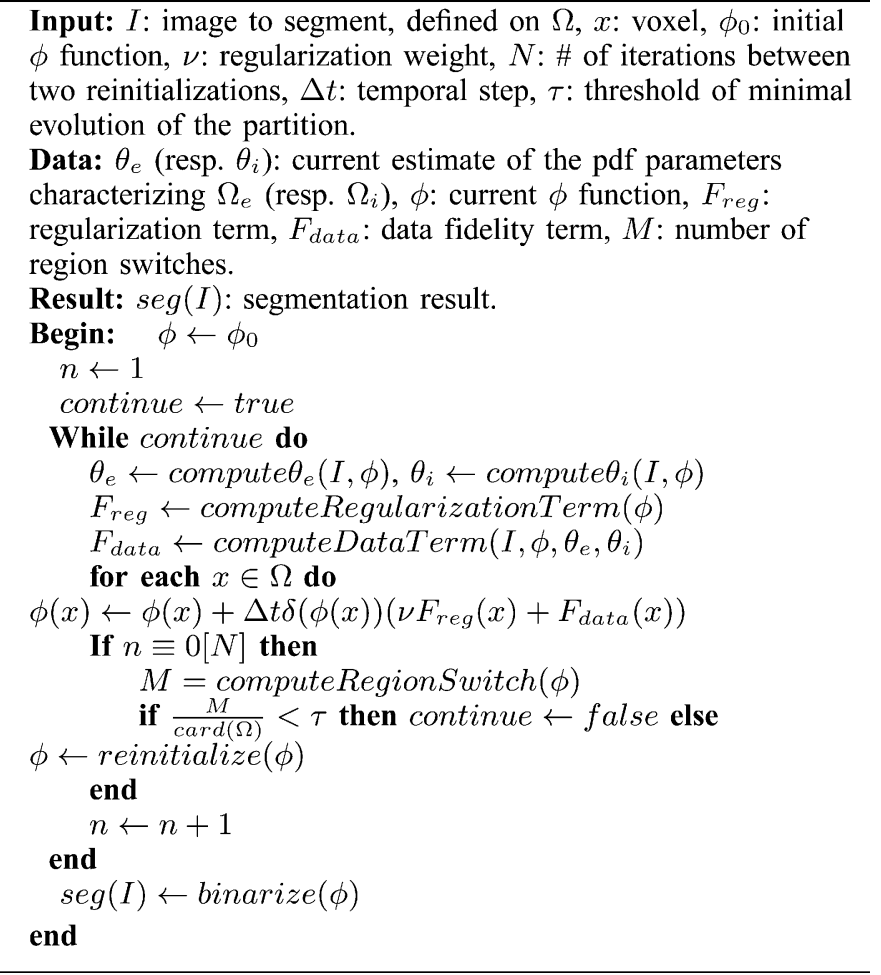

Fig. 4. Pseudocode of the segmentation algorithm.

notations for the discrete implementation of the iterative optimization process. In the implementation, the Heaviside function $H$ and Dirac function $\delta$ were approximated with smooth versions, as in [36].

1) Input Data: The $\phi_{0}$ function was generated by computing a signed distance map from a binary partition of the image, which defines the inside region $\Omega_{i}$ and the outside region $\Omega_{e}$.

The value of the regularization parameter $\nu$ was chosen empirically to obtain smooth boundaries and eliminate small connected components. This value must be set so that $F_{\text {reg }}$ and $F_{\text {data }}$ magnitudes are numerically comparable. We used $\nu=2$ for the models $R N, E N, G G$, and $\nu=4000$ for the $C V$ model since the magnitude of $F_{\text {data }}$ is much lower with $R N, E N$, and $G G$ than with $C V$.

We set $\Delta t=0.1$ in all experiments, as proposed in [41], $\tau=\frac{1}{1000}$ and $N=10$ iterations. The influence of these last two values will be discussed in the following section.

2) Numerical Evolution of the Implicit Function $\phi$ : Segmentation of the image was performed by iteratively deforming the boundary $C$ corresponding to the zero level of the level set function $\phi(x, t)$. To update the value of the implicit function at each voxel $x$, we first computed the values of the pdf parameters $\hat{\theta}_{e}$ and $\hat{\theta}_{i}$ at iteration $n+1$, denoted $\theta_{e}^{n+1}$ and $\theta_{i}^{n+1}$. We then evaluated the value of $\phi(x, n+1)$ at iteration $n+1$, from the values of $\phi(x, n)$ via the discretization and linearization of (11), using the following scheme:

$$
\begin{aligned}
\phi(x, n+1)= & \phi(x, n)+\Delta t \delta(\phi(x, n))\left(\nu F_{\text {reg }}(\phi(x, n))\right. \\
& \left.+F_{\text {data }}\left(\phi(x, n), \theta_{e}^{n+1}, \theta_{i}^{n+1}\right)\right) .
\end{aligned}
$$

The regularization force $F_{\text {reg }}(\phi(x, n))$ was discretized using the semi-implicit scheme described in [36]. The homogeneity term $F_{\text {data }}\left(\phi(x, n), \theta_{e}^{n+1}, \theta_{i}^{n+1}\right)$ was computed as the difference between $F_{i}\left(\phi(x, n), \theta_{i}^{n+1}\right)$ and $F_{e}\left(\phi(x, n), \theta_{e}^{n+1}\right)$.

3) Reinitialization of the Level Set Function and Stopping Criterion: The $\phi$ function was reinitialized every $N$ iterations, by computing the signed distance map to its zero level. The value of $N$ results from a tradeoff. On the one hand, frequent reinitializations ensure numerical stability of the spatial derivatives of $\phi$ away from the zero level. On the other hand, computation of the signed distance function is computationally expensive. By choosing $N=10$, we did not observe any degenerated behavior of the contour shape, while convergence was reached in a few hundred iterations. This empirical value was used in all experiments and did not require any fine tuning.

The segmentation algorithm stops when the partition of the image is stationary. We consider that this state is reached when the image partition does not evolve significantly between two reinitializations. Let $M$ be the number of region switches, computed as the number of voxels changing from $\Omega_{e}$ to $\Omega_{i}$ or from $\Omega_{i}$ to $\Omega_{e}$, during $N$ iterations. We consider that the solution is stationary when $M<\tau|\Omega|$. In our experiments, we used $\tau=\frac{1}{1000}, \tau$ being called the "threshold of minimal evolution of the partition" (see the following section).

4) Generation of the Final Segmentation Result: The final segmentation of $I(x)$ is defined as a binary image corresponding to the regions defined by $\phi(x, n+1)>0$ and $\phi(x, n+1)<0$. Depending on the pdf used for voxel intensity modeling, some post-processing could be needed to obtain a clean final partition of the image:

1) With the $G G$ and $C V$ models, the pdf laws are identical in $\Omega_{e}$ and $\Omega_{i}$. It is therefore necessary to determine which region corresponds to $\Omega_{\mathrm{AF}}^{*}$ (amniotic fluid in the final partition) and which region corresponds to $\Omega_{\mathrm{FT}}^{*}$ (fetal tissues in the final partition). Since the amniotic fluid is less echogenic than the fetal tissues, we rely on the mean intensities values in $\Omega_{e}$ and $\Omega_{i}$ at convergence: the region with the lowest (respectively highest) mean value is associated with $\Omega_{\mathrm{AF}}^{*}$ (respectively, $\Omega_{\mathrm{FT}}^{*}$ ).

2) With the strategies $R N$ and $E N$, the pdf laws are different in $\Omega_{e}$ and $\Omega_{i}$. Hence, an initial choice associates $\Omega_{e}$ and $\Omega_{i}$ with $\Omega_{\mathrm{AF}}^{*}$ and $\Omega_{\mathrm{FT}}^{*}$, which does not leave any room for ambiguity.

\section{B. Evaluation of the Segmentation Quality With Respect to Parameters Setting}

The iterative evolution of the segmentation process is discussed in this section, and illustrated on one 3DUS case, segmented with an $R N$ model, using a Rayleigh pdf in $\Omega_{e}$ and a Normal pdf in $\Omega_{i}$.

A slice of the 3DUS volume segmentation is illustrated in Fig. 5, with partitions $\mathcal{P}(\Omega)$ defined by $\phi(x, n)$ evolving over the iterations of the segmentation process. The evolution of the partition was fast during the first 100 iterations (phase 1), and slowed down afterward. This algorithm behavior was observed 


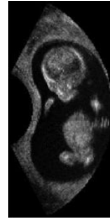

$I_{5}$

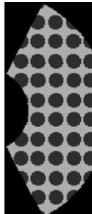

Init

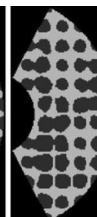

30 it.

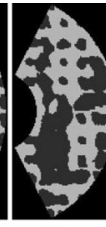

$60 \mathrm{it}$

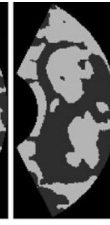

90 it.

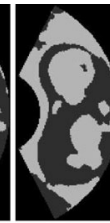

120 it.

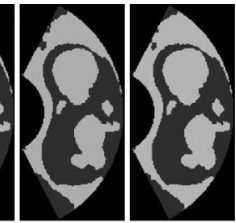

220 it. 390 it.
Fig. 5. Iterative segmentation of one 3DUS volume. One slice of the 3DUS volume is presented along with the regions $\Omega_{e}$ and $\Omega_{i}$ at initialization and after $30,60,90,120,220$, and 390 iterations of the minimization of the energy functional.

on all the 3DUS volumes that were processed, for all the pdf models.

We evaluated the influence of the stopping criterion. For the example shown in Fig. 5, with $\tau=\frac{1}{500}, \tau=\frac{1}{1000}$, and $\tau=\frac{1}{2000}$, the algorithm converged at iterations 120, 220, and 390, respectively. This illustrates the impact of the tradeoff between the numbers of iterations and the final segmentation quality. Images in Fig. 5 illustrate the corresponding final segmentation results, which are almost identical. If we look in more details, we observe that with $\tau=\frac{1}{500}$, the uterine wall and the embryo's head are not fully closed. By using $\tau=\frac{1}{1000}$, local improvements of $\mathcal{P}(\Omega)$ were obtained and the previous segmentation defects were no longer observed. Finally, very little differences were observed with $\tau=\frac{1}{2000}$, despite 100 additional iterations. The value $\tau=\frac{1}{1000}$ therefore seems to be a suitable intermediate value, for which the partition reaches a suitable stability. This was the case for all volumes of the database. This parameter can be interpreted as follows: $\tau$ encodes the authorized proportion of image points that can oscillate between phases while considering the partition to be stabilized. Using a $\tau=1 / 1000$ means that convergence is considered when less than $0.1 \%$ of the points in the image change phases between few iterations. The points that keep on oscillating at convergence of the partition are points at the interface between two phases. In the absence of a priori knowledge on the perimeter of the object to segment, the usual approach is to perform empirical tests to derive a generic value for $\tau$, suited for the type of image data to segment, that provides a good tradeoff between the number of iterations needed to converge and the final segmentation quality. We followed this approach, and tests were performed on the whole database with similar results, leading to this value of $\tau=1 / 1000$.

\section{Analysis of Homogeneity Measures in $F_{D a t a}$}

We illustrate in Fig. 6 the different profiles of the homogeneity measures $F_{e}, F_{i}$ and the energy term $F_{\text {data }}$ as a function of voxel intensities, at convergence of the iterative segmentation process, considering the models $S D(E N)$ (a), $G G$ (b), and $C V$ (c). Note that the figure is related to the final segmentation result, and not the curve evolution. These profiles were computed for one case to illustrate the fact that the implicit threshold value depends on the modeling strategy. Note that it would not be suited to display profiles merged from different cases, since they do not have calibrated values, and therefore, parameters of the distribution functions vary between cases, as well as the final implicit threshold, defined below.
A voxel of intensity $I(x)$ is more likely to belong to $\Omega_{e}$ (respectively, $\Omega_{i}$ ) if $F_{\text {data }}$ is negative (respectively, positive). The profile of $F_{\text {data }}$ therefore defines an implicit threshold value $s^{m}$ for the model $m$, corresponding to its zero crossing, i.e. the intensity value for which $F_{\text {data }}=0$. At this value, indicated by the black-dotted line in the figure, $F_{e}$ and $F_{i}$ are equal, and $\Omega_{i}$ and $\Omega_{e}$ equally probable. This implicit threshold value separates the two phases of the image partition.

We can observe that the profile of $F_{\text {data }}$ depends on the modeling strategy and different implicit threshold values are obtained for each model. A 2-D slice of a clinical case example is shown in Fig. 7(a). The hypoechogenic class in this image is composed of the placenta (green ellipse), the fetus' skull content (red ellipse) degraded by an acoustic shadow due to a strong reflexion on the skull bone, and the amniotic fluid, part of which is located behind the fetus' trunk (blue ellipse). The influence of the model and its final implicit threshold value defined by $F_{\text {data }}$ is visible on the segmentation results in Fig. 7(b)-(d), obtained with $E N(\mathrm{~b}), G G$ (c), and $C V$ (d) models.

In this example, the lowest threshold value was obtained with the $E N$ model (where $s^{E N}=41$ ). This value enabled to segment properly the placenta and the fetus' skull, but the amniotic fluid region located behind the fetus was segmented as belonging to fetal tissues. For the $C V$ model, the highest threshold value was obtained $\left(s^{C V}=72\right)$, and the amniotic fluid region was correctly segmented (d). However, the segmentation of the placenta and the fetal skull were not satisfying, as voxels intensities are low in these structures. With the $G G$ model $\left(s^{G G}=50\right)$, we obtained a reasonable tradeoff between the two other partitions (c).

Moreover, Fig. 6 illustrates the fact that the magnitude of $F_{\text {data }}$ depends on the pdf exploited by the different models. For voxel intensities within the range $[0,255]$ in the database, $F_{\text {data }}$ varies in an interval of values of width 20 with the $R N, E N$, and $G G$ models, and in an interval of values of width 40000 with the $C V$ model. This observation was used to set the value of $\nu$, as mentioned in Section IV-A, leading to an appropriate weighting of the regularization energy term $F_{\text {reg }}$ in the global energy functional.

\section{Robustness of Segmentation Results With Respect to the Initialization}

To evaluate the robustness of the proposed segmentation method with respect to the initial partition, three initialization strategies, denoted $S M, S O$, and $C Y$, were tested to define $\phi_{0}$. The $\phi_{0}$ function was computed as the signed distance from the contours $C_{0}$ of a binary image. The binary images correspond to initial guesses of the image partition into two classes.

The binary images used to build $S M$ and $S O$ were obtained via intensity thresholding, considering learned threshold values, from the manual thresholding experiments presented in Section II-A. When processing an image $I_{i}$ from the database, the threshold value for $S M$ was defined as $\mu_{i}^{s}$ which corresponds to the average threshold value defined by the expert (computed on the whole database except $I_{i}$ ). The threshold value for $S O$ was defined as $o_{i}$, which corresponds to the K-means threshold 


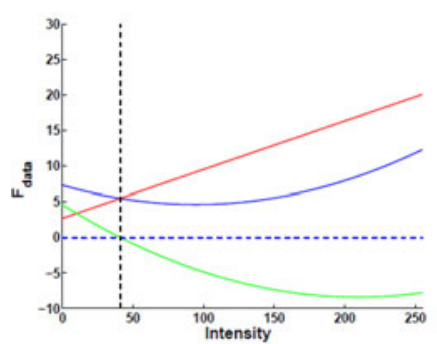

(a)

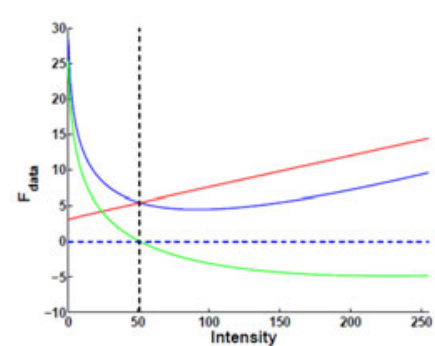

(b)

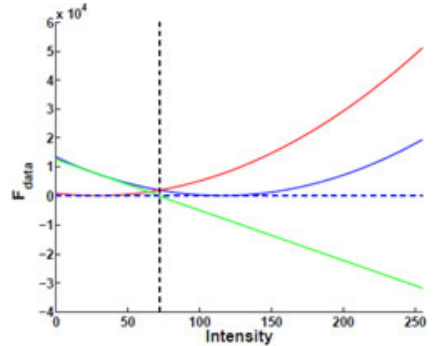

(c)

Fig. 6. Profiles of homogeneity measures $F_{e}$ (red), $F_{i}$ (blue), and $F_{\text {data }}$ (green) at convergence of the segmentation process, for one 3DUS volume, using (a) $E N$, (b) $G G$, and (c) $C V$ models. The black-dotted line identifies the implicit threshold value, obtained as the intensity for which $F_{\text {data }}=0$, and which depends on the modeling strategy.

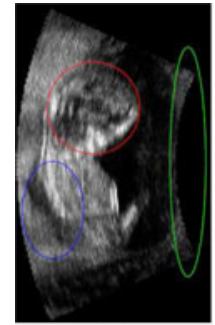

(a)

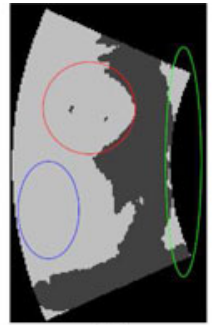

(b)

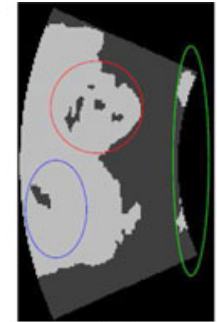

(c)

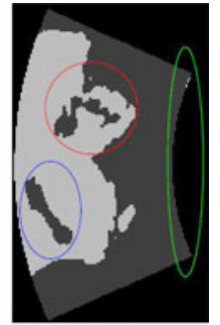

(d)

Fig. 7. Example of segmentation results with different models on a 2-D slice from one 3DUS volume. (a) Slice from the 3DUS volume and corresponding segmentation results for the (b) $E N$, (c) $G G$, and (d) $C V$ models. Regions in the placenta (green ellipse), the fetus' skull content (red ellipse) degraded by an acoustic shadow due to a strong reflexion on the skull bone, and the amniotic fluid, part of which is located behind the fetus' trunk (blue ellipse) are segmented differently.

computed on image $I_{i}$. The binary image with the $C Y$ initialization was not defined from an a priori threshold value, but simply as a set of cylinders partitioning the image into two classes as in [36]. This corresponds to a traditional initial geometric configurations of $\phi_{0}$ when no a priori information is available.

To evaluate the robustness of the proposed segmentation method with respect to initial conditions, overlap and similarity measures between the partitions obtained with the different initialization strategies and the different models described in Table I were computed. Let $m \in\{S D, G G, C V\}$ be the model and $j \in\{S M, S O, C Y\}$ the initialization strategy, the segmentation result obtained on image $I_{i}$, using $m$ and $j$, is denoted $\operatorname{seg}_{i}^{m}(j)$.

The overlap measure $\operatorname{rec}_{i}^{m}(j, k)$ for a given model $m$ is defined as $\operatorname{rec}_{i}^{m}(j, k)=\frac{\left|\operatorname{seg}_{i}^{m}(j) \cap \operatorname{seg}_{i}^{m}(k)\right|}{\left|\operatorname{seg}_{i}^{m}(j) \cup \operatorname{seg}_{i}^{m}(k)\right|}, j \neq k$. The minimum overlap value over the different pairs of initializations $(j, k)$ is denoted $r e c_{i}^{m}$ and corresponds to the less similar results. Over the whole database, the $\mu_{r e c}^{m}, \min _{r e c}^{m}, \max _{r e c}^{m}$ measures denote the mean, minimum, and maximum values of the minimum overlap for a given modeling strategy $m$ (i.e., the mean, minimum, and maximum values over $i$ of $\left\{\operatorname{rec}_{i}^{m}\right\}$ ). We obtained average values $\mu_{r e c}^{S D}=0.92, \mu_{\text {rec }}^{G G}=0.93$, and $\mu_{r e c}^{C V}=0.93$, min values of $\min _{r e c}^{S D}=0.86, \min _{r e c}^{G G}=0.89$, and $\min _{r e c}^{C V}=0.88$ and $\max$ values of $\max _{r e c}^{S D}=0.96, \max _{\text {rec }}^{G G}=0.96$, and $\max _{r e c}^{C V}=0.96$. The mean overlap values computed on the less similar partitions were high for all modeling strategies, confirming a good

robustness of the proposed segmentation method with respect to initial conditions. We also compared the implicit threshold values corresponding to the different segmentation setups. Let $s_{i}^{m}(j)$ be the implicit threshold defined by the homogeneity term $F_{\text {data }}$ at convergence of the segmentation process for the image $I_{i}$, using the model $m$ and the initialization $j$. Let $\epsilon_{i}^{m}(j, k)=\left|s_{i}^{m}(j)-s_{i}^{m}(k)\right|$, with $j \neq k$, be the difference between the thresholds $s_{i}^{m}(j)$ and $s_{i}^{m}(k)$. Let $\epsilon_{i}^{m}$ be the maximum difference of the $\epsilon_{i}^{m}(j, k)$ values over the different pairs of initializations $(j, k)$. We denote by $\mu_{\epsilon}^{m}$ and $\sigma_{\epsilon}^{m}$ the mean and the standard deviation of the $\epsilon_{i}^{m}$ measures over the database. For the different modeling strategies, we measured $\mu_{\epsilon}^{S D}=5.7, \mu_{\epsilon}^{G G}=$ 4.1, $\mu_{\epsilon}^{C V}=3$ and $\sigma_{\epsilon}^{S D}=4, \sigma_{\epsilon}^{G G}=2.5, \sigma_{\epsilon}^{C V}=2.1$. Comparing average manual (intra-observer) and automated variability of the threshold values, we obtained that $\epsilon_{\mu}^{m}<\mu_{\epsilon}$ and $\sigma_{\epsilon}^{m}<\sigma_{\epsilon}$ for all the modeling strategies, since the intra-observer variability was measured as $\mu_{\epsilon}=12.8$ and $\sigma_{\epsilon}=5$.6. Moreover, the differences between the threshold values obtained with our automated approach were systematically smaller than those obtained with the expert. This analysis, therefore, confirms that the threshold values obtained with the proposed segmentation approach are less variable than those defined by a human expert. This stresses that the method exhibits a satisfactory robustness with respect to the initialization, based on this reproducibility criterion.

To homogenize the evaluation conditions in the following, we will only consider the result obtained with the $S M$ initialization, which appeared to be appropriate for all cases. Moreover, we experimentally observed that the convergence was fastest with this initialization. We denote in the following $\operatorname{seg}_{i}^{m}=\operatorname{seg}_{i}^{m}(S M)$.

\section{E. Quantitative Evaluation of the Segmentation Results Accuracy}

Segmentation results obtained automatically were compared with manual segmentations. While segmentations were always performed in 3-D, quantitative evaluation was performed as follows: in 3-D for five clinical case from the database for which a detailed 3-D manual segmentation was available, and in 2-D on the other clinical cases for which a detailed manual segmentation was only available on three orthogonal slices. The manual segmentation result of a volume $I_{i}$ is denoted $\operatorname{seg}_{i}^{M A N}$. Similarity between the manual segmentation $\operatorname{seg}_{i}^{M A N}$ and the automated segmentation $\operatorname{seg}_{i}^{m}$ was evaluated by computing two measures: the overlap between regions and the mean distance (expressed 


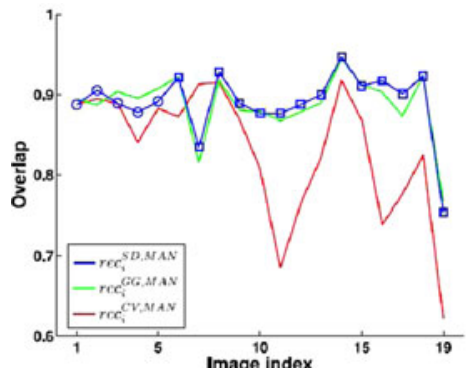

(a)

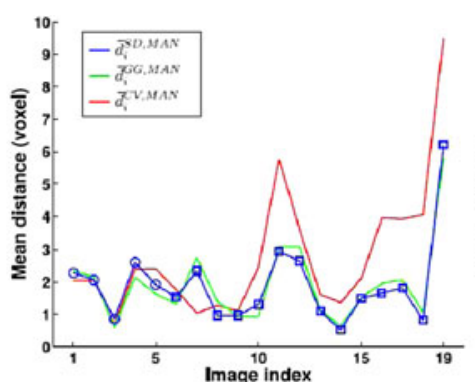

(b)

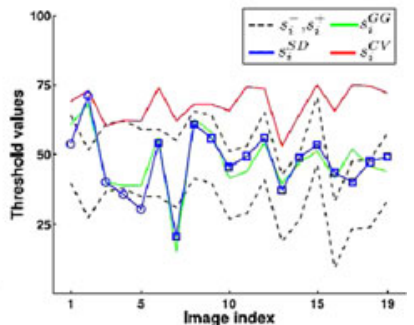

(c)

Fig. 8. Evaluation, on the 3DUS images, of the similarities between the automated segmentation and the manual detailed segmentation. (a) Overlap measure $\operatorname{rec}_{i}^{m, M A N}$ and (b) mean tissue interfaces distance $\bar{d}_{i}^{m, M A N}$ for the automated segmentations obtained with the model $m$. The blue curves correspond to $S D$, the green ones to $G G$, and the red ones to $C V$. Squares correspond to $S D=E N$, and circles to $S D=R N$. (c) Implicit threshold values defined by the homogeneity measure $F_{\text {data }}: s_{i}^{S D}$ (blue), $s_{i}^{G G}$ (green), and $s_{i}^{C V}$ (red), along with the manually defined extremal admissible threshold values plotted with the two black-dotted curves.

in voxels) between tissue interfaces, denoted $\operatorname{rec}_{i}^{\text {m, MAN }}$ and $\bar{d}_{i}^{m, M A N}$, respectively. The mean distance (based on the L2 norm) compares binary volumes at the voxel level, without extracting a surface, and is usually more informative than the Hausdorff distance, since US images are noisy and intratracer variability is very high. The Hausdorff distance is suited when a precise delineation is targeted, and comparison is made to a precise ground truth. In [24], for example, the authors only used the mean distance to compare segmentations of fetal faces, seeking clinical evaluation of their segmentation tool. In clinical studies, such as in [42], only correlations of specific biometric measures are studied.

Similarity measures are plotted in Fig. 8(a) and (b). Let $r e c^{m, M A N}$ and $\bar{d}^{m, M A N}$ be the average values of the overlap and distance measures on the database, considering the model $m$. We obtained the following average values: $\operatorname{rec}^{S D, M A N}=$ $0.89, \operatorname{rec}^{G G, M A N}=0.89, \operatorname{rec}^{C V, M A N}=0.83$ and $\bar{d}^{S D, M A N}=$ $1.8, \bar{d}^{G G, M A N}=1.9, \bar{d}^{C V, M A N}=2.7$ (distances are given in voxels). We observed that results obtained with the modeling strategies exploiting statistical distributions $S D$ and $G G$ were very similar, and superior to those obtained with the $C V$ model, which did not compare well with the manual reference for several cases.

We also evaluated the accuracy of the segmentation in terms of identifying an implicit threshold close to the manual threshold value. Fig. 8(c) reports the $s_{i}^{m}$ values for the different modeling strategies, together with the minimum and maximum manually defined admissible values. One can note that the $s_{i}^{S D}$ and $s_{i}^{G G}$ are within the range of the admissible values for most images, while it is the case for only two images for the $s_{i}^{C V}$ values. More precisely, threshold values defined with the $C V$ model are too high for many images. Voxels belonging to $\Omega_{\mathrm{FT}}$ and located in regions affected by acoustic shadows, posterior reinforcement or Total Gain Compensation artifacts have abnormally low intensities. If the implicit threshold defined by the homogeneity term $F_{\text {data }}$ is high, these voxels will be associated with $\Omega_{\mathrm{AF}}^{*}$ in the final partition. This behavior, illustrated in Fig. 7(d), explains the lower quality of the results (regarding overlap and distance measures) obtained when the $C V$ model is considered. On the other hand, the $G G$ and $S D$ models provided overall very similar quantitative measures of segmentation quality.

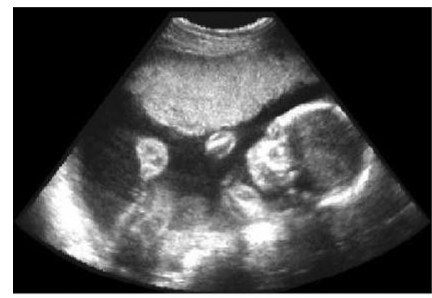

(a)

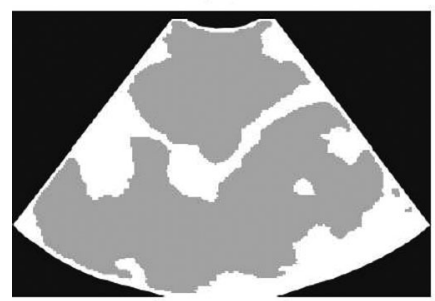

(c)

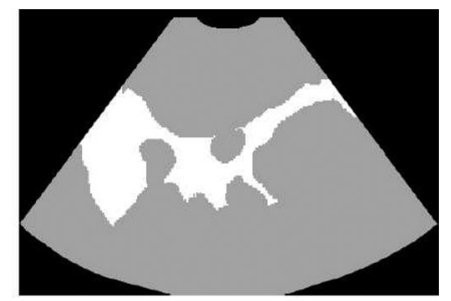

(b)

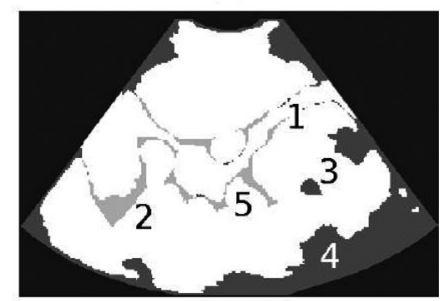

(d)
Fig. 9. Illustration of segmentation discrepancies on the case with the largest segmentation error measures (acquired at $22 \mathrm{WA}$ ). (a) Single slice from the 3DUS volume $I_{19}$. Segmentation results (b) $\operatorname{seg}_{19}^{M A N}$ and (c) $\operatorname{seg}_{19}^{S D}$. Voxels of $\Omega_{\mathrm{AF}}$ and $\Omega_{\mathrm{AF}}^{*}$ are displayed in white, while voxels of $\Omega_{\mathrm{FT}}$ and $\Omega_{\mathrm{FT}}^{*}$ are displayed in gray. (d) Segmentation differences between $\operatorname{seg}_{19}^{M A N}$ and $\operatorname{seg}_{19}^{S D}$. Numbers in (d) refer to the sources of errors discussed in the text.

Beyond global segmentation accuracy evaluation, we also analyzed local segmentation errors. The interface between the amniotic fluid and the fetal tissues is generally slightly blurred, which makes the manual segmentation difficult at the voxel level, leading to small segmentation discrepancies around this boundary. But otherwise the results are very satisfactory. For all 18 first trimester cases, automated segmentation results were visually very similar to the manual ones, as confirmed by the quantitative evaluation measures. Fig. 9 illustrates the segmentation results for a single slice of the clinical case $I_{19}$, acquired at 22 WA using the iU22 transducer, showing $\operatorname{seg}_{19}^{M A N}$ (the reference) and $\operatorname{seg}_{19}^{S D}$, and the difference image between them. On the difference image, white voxels are correctly segmented, gray pixels correspond to segmentation errors, and black pixels are out of the field of view. Light (respectively, dark) gray voxels are classified in $\Omega_{\mathrm{FT}}^{*}\left(\Omega_{\mathrm{AF}}^{*}\right)$ in $\operatorname{seg}_{19}^{S D}$ while they belong to $\Omega_{\mathrm{AF}}$ $\left(\Omega_{\mathrm{FT}}\right)$ in the reference $\operatorname{seg}_{19}^{M A N}$. Although it is difficult to compare the results with those obtained on the other data since the 


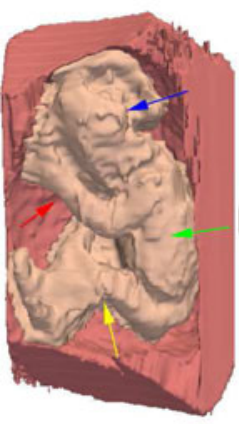

(a) (b)

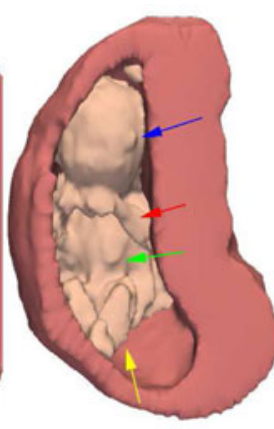

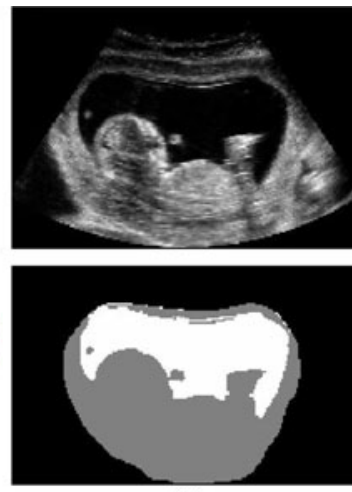

(c)
Fig. 10. Illustration of the 3-D reconstructions of fetal tissues corresponding to (a) $\operatorname{seg}_{19}^{S D}$ and (b) $\operatorname{seg}_{18}^{G G}$ for anatomical modeling of pregnant women. The placenta is displayed in red and the fetus is displayed in pink. The arrows correspond to the fetus skull, torso, legs, and arms. The images on the right (c) illustrate a single slice of the 3DUS volume $I_{18}$ and the corresponding segmentation result $\operatorname{seg}_{18}^{G G}$. Results for $I_{19}$ are displayed in Fig. 9.

transducer is not the same and the resolution is lower, it can be observed that this case at 22 WA generated significantly lower segmentation accuracy, when compared to manual segmentation. This lower performance of a binary partition of the images into two homogeneous tissue types could be expected, whatever the acquisition setup, since this case is out of the range of the first trimester of gestation. Indeed, illustration of the segmentation results on the case acquired at 22 WA shows the limits of the proposed approach when the image displays more complex fetal structures and more shadowing artifacts generated by calcified bones. The binary partition model is no longer suited when the image gets more complex, with more visible fetal structures (but again the results for all other cases are good, as illustrated, for instance, in Fig. 10). Visual analysis of the automated segmentation results allows us to understand the different phenomena causing the large segmentation discrepancies observed in Fig. 9(d). It appears that the main causes of errors are due to 1) the blurred interface between the amniotic fluid and the fetal tissues; 2) the introduction of artificially high intensity values during the image interpolation process from the acquisition grid to a Cartesian grid; 3) the presence of large acoustic shadows; 4) the low intensity of the voxels located far from the transducer despite Total Gain Compensation; and 5) the effect of the regularization term $F_{\text {reg }}$ which prevents the proper segmentation of strong concavities. However, despite these errors, the morphology of the fetus is preserved and can be well distinguished, as illustrated in Fig. 10. Finally, the proposed segmentation framework enabled to partition automatically 3DUS volumes englobing the whole embryo. Little user interactions were required to separate the uterus from the fetal tissues. We illustrate in Fig. 10 two 3-D reconstructions generated from two segmentation results.

\section{DISCUSSION AND SUMMARY}

The segmentation method proposed in this paper aims at extracting automatically two types of tissues on fetal 3DUS images: fetal tissues and amniotic fluid. This problem was previ- ously addressed only partially and in few works. To guide the segmentation process, we tested several combinations of statistical distribution models of the voxel intensities in the two types of tissues. Histograms of the voxel intensities differ greatly in the fetal tissues and the amniotic fluid, and depend on the image saturation. We therefore proposed specific distributions for each type of tissue and for saturated/unsaturated images. For saturated images, we proposed to combine Exponential and Normal distributions, while for non-saturated images, we proposed to use Rayleigh and Normal distributions. A more generic formulation of the pairs of models with the Gamma distribution was also studied, covering all situations. The segmentation problem was formulated as the minimization of an energy functional. The energy was itself derived from a Bayesian MAP formulation of the partition of the image into "homogeneous" regions. Homogeneity of the regions was quantified via probability measures exploiting the statistical distribution models. Energy minimization was implemented using the level-set formalism and an iterative estimation of the optimal partition via gradient descent. We proposed a generic expression of the segmentation problem in order to compare the proposed method with the one described in [36], where voxel intensities within the regions are modeled with constant intensity values.

Since the optimization process does not guarantee to obtain a partition of the image corresponding to a global minimum of the energy, we first evaluated the robustness of the segmentation process with respect to the initial conditions (i.e., the initial partition of the image). Evaluation results showed that the method is little sensitive to initialization conditions. Average overlap values in Section IV-D provide a level of intrinsic variability of a given modeling strategy with different initializations. They can, therefore, be seen as cap values of segmentation precision achievable by a given segmentation method. The different modeling strategies were evaluated in terms of segmentation accuracy comparing to manual segmentation. All models exploiting statistical distributions of the voxel intensities provided similar segmentation accuracy, and proved to be more accurate than the piecewise constant model from [36]. This shows the interest of using adaptive distributions, depending on the image characteristics and the type of tissue to segment. This approach contrasts with other methods that blindly use Rayleigh distributions, or distributions learned from manually delineated images which are then used to segment other images (e.g., [43] for prostate segmentation), or distributions that depend on the segmentation objective but not on individual images (for instance, the authors in [37] use Rayleigh distributions to segment the left ventricle and the classical Chan-Vese model to segment the prostate). Evaluations were performed on a database of 19 3-D images, acquired in standard clinical conditions, and exhibiting great variability in terms of fetus ages, acquisition setup, and image properties. This constitutes thus a good preliminary validation, which is promising for further large-scale studies. We can expect a reasonable robustness with respect to image characteristics and acquisition conditions, since the proposed segmentation method uses tissue appearance models that are adapted to the type of US image acquisition (saturated or not), the type of tissue to extract, and the tissue appearance via 
optimization of the modeling parameters, and since segmentation performances were stable for 19 cases, acquired in clinical routine and not optimized for this particular study. More extensive clinical validation could be based on several manual segmentations, provided by different experts, so that inter-expert variability could be assessed. A next step could also be to provide a methodology to decide a priori on the best modeling strategy.

Our segmentation method was implemented in MATLAB, without code optimization. An order of magnitude of 20 minutes for a $100 \times 200 \times 100$ matrix size was needed for the whole process. The only hint to reduce running time, besides code optimization, is to start with a good initialization, hence reducing the number of iterations.

Three-dimensional reconstructions, based on the segmentation results, were illustrated and enabled to precisely visualize the fetus in the uterine environment, even on challenging images with strong artifacts.

The segmentation results provided by our method were interactively refined by distinguishing the fetus body, the umbilical cord, and the placenta. Since the contact region between the fetus and the uterine wall is small, little user interaction was required to perform this task. In a clinical context, the VOCAL software tool can be used to interactively segment the amniotic sac as proposed in [44], with limited effort thanks to the powerful visualization and interaction functionalities of this tool. Echographers are getting familiar with the manipulation of such interactive segmentation tools for 3DUS images but still consider a full segmentation to be cumbersome in clinical routine. Future methodological developments will consist in identifying the three aforementioned structures, in an automated or semiautomated fashion, for applications such as biometric measures (amniotic sac and fetal volumes, crown-rump length, etc.) on large datasets.

Morphological and biometrical analysis of the fetus could be performed using the proposed automated segmentation tool. There are several potential clinical applications for the exploitation of detailed segmentations of the fetus during the first trimester: detection of asymmetries between the cephalic pole and the embryo's body is of interest to identify severe abnormalities and identify potential correlation with miscarriage probability; quantification of fetus volumes could be exploited to assess fetal development and refine delivery date prediction; finer image markers of abnormalities could be investigated, exploiting the automated localization of the ocular lobes, the limbs, the stomach, the bladder, and the nuchal translucency; comparison of 3-D measures with 1-D/2-D measurements extracted from standard 2-D echographic images is still needed to evaluate the clinical impact of 3DUS imaging for biometry.

Our study was performed in the context of anatomical modeling of pregnant women for numerical dosimetry simulations. In this context, we exploited four 3DUS cases to generate detailed anatomical models used to investigate the influence of electromagnetic fields on the fetus during pregnancy (see [45] and [46] for details). Surfacic models of the uterus and its content were built and embedded within a synthetic woman model. These models were manually refined by distinguishing, from the auto-

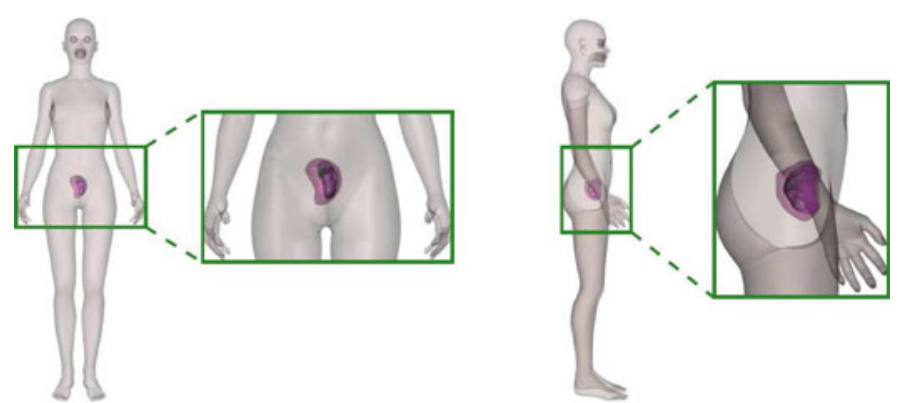

Fig. 11. Pregnant woman model at $15 \mathrm{WA}$, embedding the utero-fetal unit segmented on a 3DUS clinical case. Coronal and sagittal views of the 3-D model are provided.

mated segmentation results, the fetus body envelope, the umbilical cord, and the placenta. An example of a generated pregnant woman model at $13 \mathrm{WA}$ is provided in Fig. 11. Additional pregnant woman models are publically available for download for research purposes on the web page of the FEMONUM project: http://femonum.telecom-paristech.fr/.

Future developments will focus on preprocessing US images to attenuate strong artefacts and on the refinement of the segmentation process to identify, in an automated or semi-automated fashion, internal fetal organs (brain, heart), as well as the three aforementioned structures, for instance, using a similar methodology but in a multiphase framework [47].

\section{ACKNOWLEDGMENT}

The authors would like to thank the members of Prof. D. Lutons' group (Department of Obstetrics and Gynecology, Beaujon Hospital, Clichy, France) and Philips Medical Research Lab (Suresnes, France) for providing the 3-D ultrasound data.

\section{REFERENCES}

[1] M. B. McNay and J. E. E. Fleming, "Forty years of obstetric ultrasound 1957-1997: From A-scope to three dimensions," Ultrasound Med. Biol., vol. 25 , no. 1 , pp. 3-56, 1999.

[2] P. Callen, Ultrasonography in Obstetrics and Gynecology. Philadelphia, PA: Saunders, 1988.

[3] N. Roberts and B. Thilaganathan, "The role of ultrasound in obstetrics," Obst. Gynaecol. Reproduct. Med., vol. 17, no. 3, pp. 79-85, 2007.

[4] N. Dudley, "A systematic review of the ultrasound estimation of fetal weight," Ultrasound Obst. Gynecol., vol. 25, no. 1, pp. 80-89, 2005.

[5] R. Rankin, A. Fenster, D. Downey, P. Munk, M. Levin, and A. Vellet, "Three-dimensional sonographic reconstruction: Techniques and diagnostic applications," Amer. J. Roentgenol., vol. 161, no. 4, pp. 695-702, 1993

[6] L. Merce, M. Barco, and S. Bau, "Three-dimensional volume sonographic study of fetal anatomy: Intraobserver reproducibility and effect of examiner experience," J. Ultrasound Med., vol. 27, no. 7, pp. 1053-1063, 2008.

[7] M. Odeh, Y. Hirsh, S. Degani, V. Grinin, E. Ofir, and J. Bornstein, "Threedimensional sonographic volumetry of the gestational sac and the amniotic sac in the first trimester," J. Ultrasound Med., vol. 27, no. 3, pp. 373-378, 2008.

[8] H. Blaas, P. Taipale, H. Torp, and S. Eik-Nes, "Three-dimensional ultrasound volume calculations of human embryos and young fetuses: A study on the volumetry of compound structures and its reproducibility," Ultrasound Obst. Gynecol., vol. 27, no. 6, pp. 640-646, 2006.

[9] O. Falcon, C. Peralta, P. Cavoretto, S. Faiola, and K. Nicolaides, "Fetal trunk and head volume measured by three-dimensional ultrasound at $11+$ 0 to $13+6$ weeks of gestation in chromosomally normal pregnancies," Ultrasound Obst. Gynecol., vol. 26, no. 3, pp. 263-266, 2005.

[10] C. Peralta, P. Cavoretto, B. Csapo, O. Falcon, and K. Nicolaides, "Lung and heart volumes by three-dimensional ultrasound in normal fetuses at 12-32 
weeks gestation," Ultrasound Obst. Gynecol., vol. 27, no. 2, pp. 128-133, 2006.

[11] N. Raine-Fenning, J. Clewes, N. Kendall, A. Bunkheila, B. Campbell, and I. Johnson, "The interobserver reliability and validity of volume calculation from three-dimensional ultrasound datasets in the in vitro setting," Ultrasound Obst. Gynecol., vol. 21, no. 3, pp. 283-291, 2003.

[12] B. Tutschek and D. Sahn, "Semi-automatic segmentation of fetal cardiac cavities: Progress towards an automated fetal echocardiogram," Ultrasound Obst. Gynecol., vol. 32, no. 2, pp. 176-180, 2008.

[13] A. Noble and D. Boukerroui, "Ultrasound image segmentation: A survey," IEEE Trans. Med. Imag., vol. 25, no. 8, pp. 987-1010, Aug. 2006.

[14] J. Thomas, R. Petters, and P. Jeanty, "Automatic segmentation of ultrasound images using morphological operators," IEEE Trans. Med. Imag., vol. 10, no. 2, pp. 180-186, Jun. 1991.

[15] G. Matsopoulos and S. Marshall, "Use of morphological image processing techniques for the measurement of a fetal head from ultrasound images," Pattern Recognit., vol. 27, no. 10, pp. 1317-1324, 1994.

[16] M. Kass, A. Witkin, and D. Terzopoulos, "Snakes: Active contour models," Int. J. Comput. Vis., vol. 1, no. 4, pp. 321-331, 1988.

[17] V. Chalana, T. Winter 3rd, D. Cyr, D. Haynor, and Y. Kim, "Automatic fetal head measurements from sonographic images," Acad. Radiol., vol. 3 , no. 8, pp. 628-635, 1996.

[18] S. Jardim and M. Figueiredo, "Segmentation of fetal ultrasound images," Ultrasound Med. Biol., vol. 31, no. 2, pp. 243-250, 2005.

[19] G. Carneiro, B. Georgescu, S. Good, and D. Comaniciu, "Detection and measurement of fetal anatomies from ultrasound images using a constrained probabilistic boosting tree," IEEE Trans. Med. Imag., vol. 27, no. 9, pp. 1342-1355, Sep. 2008.

[20] A. Sarti, R. Malladi, and J. Sethian, "Subjective surfaces: A geometric model for boundary completion," Int. J. Comput. Vis., vol. 46, no. 3, pp. 201-221, 2002.

[21] I. Dindoyal, T. Lambrou, J. Deng, C. Ruff, A. Linney, and A. ToddPokropek, "Level set segmentation of the foetal heart," in Functional Imaging and Modeling of the Heart Proceedings. New York: SpringerVerlag, 2005, pp. 201-221.

[22] A. Sarti, C. Corsi, E. Mazzini, and C. Lamberti, "Maximum likelihood segmentation of ultrasound images with Rayleigh distribution," IEEE Trans. Ultrason., Ferroelectr., Freq. Control, vol. 52, no. 6, pp. 947-960, Jun. 2005.

[23] M. Sofka, J. Zhang, S. Zhou, and D. Comaniciu, "Multiple object detection by sequential Monte Carlo and hierarchical detection network," in Proc. IEEE Conf. Comput. Vis. Pattern Recognit., Jun. 2010, pp. 1735-1742.

[24] S. Feng, S. Zhou, S. Good, and D. Comaniciu, "Automatic fetal face detection from ultrasound volumes via learning 3-D and 2-D information," in Proc. IEEE Conf. Comput. Vis. Pattern Recognit., Jun. 2009, pp. 2488 2495.

[25] G. Carneiro, F. Amat, B. Georgescu, S. Good, and D. Comaniciu, "Semantic-based indexing of fetal anatomies from 3-D ultrasound data using global/semi-local context and sequential sampling," in Proc. IEEE Conf. Comput. Vis. Pattern Recognit., Jun. 2008, pp. 1-8.

[26] N. Otsu, "A threshold selection method from gray-level histograms," $\mathrm{A} u$ tomatica, vol. 11, pp. 285-296, 1975.

[27] J. Hartigan and M. Wong, "A k-means clustering algorithm," Appl. Statist., vol. 28 , no. 1, pp. 100-108, 1979.

[28] S. Choi and R. Wette, "Maximum likelihood estimation of the parameters of the gamma distribution and their bias," Technometrics, vol. 11, no. 4 pp. 683-690, 1969.

[29] Z. Tao, H. Tagare, and J. Beaty, "Evaluation of four probability distribution models for speckle in clinical cardiac ultrasound images," IEEE Trans. Med. Imag., vol. 25, no. 11, pp. 1483-1491, Nov. 2006

[30] R. Wagner, S. Smith, J. Sandrik, and H. Lopez, "Statistics of speckle in ultrasound B-scans," IEEE Trans. Sonics Ultrason., vol. SU-30, no. 3, pp. 156-163, May 1983.
[31] C. Burckhardt, "Speckle in ultrasound B-mode scans," IEEE Trans. Sonics Ultrason., vol. SU-25, no. 1, pp. 1-6, Jan. 1978.

[32] N. Paragios, M. Jolly, M. Taron, and R. Ramaraj, "Active shape models and segmentation of the left ventricle in echocardiography," in Proceedings of the International Conference on Scale Space Theories PDEs Methods Computer Vision. New York: Springer-Verlag, 2005, pp. 131-142.

[33] M. Hansen, J. Moller, and F. Togersen, "Bayesian contour detection in a time series of ultrasound images through dynamic deformable template models," Biostatistics, vol. 3, no. 2, pp. 213-228, 2002.

[34] F. Shao, K. Ling, and W. Ng, "Automatic 3-D prostate surface detection from TRUS with level sets," Int. J. Imag. Graph., vol. 4, no. 3, pp. 385403, 2004.

[35] J. Durbin and M. Knott, "Components of Cramer-von Mises statistics. I," J. Royal Statist. Soc. Ser. B, vol. 34, pp. 290-307, 1972.

[36] T. Chan and L. Vese, "Active contours without edges," IEEE Trans. Imag. Process., vol. 10, no. 2, pp. 266-277, Feb. 2001

[37] C. Ahn, Y. Jung, O. Kwon, and J. Seo, "A regularization technique for closed contour segmentation in ultrasound images," IEEE Trans. Ultrason., Ferroelectr., Freq. Control, vol. 58, no. 8, pp. 1577-1589, Aug. 2011.

[38] S. Osher and J. Sethian, Fronts Propagating With Curvature Dependent Speed: Algorithms Based on Hamilton-Jacobi Formulations. Hampton, VA: NASA Langley Res. Center, 1987.

[39] S. Osher and N. Paragios, Geometric Level Set Methods in Imaging, Vision, and Graphics. New York: Springer-Verlag, 2003.

[40] M. Rousson and R. Deriche, "A variational framework for active and adaptive segmentation of vector valued images," in Proc. IEEE Workshop Motion Video Comput., Dec. 2002, pp. 56-62.

[41] L. Vese and T. Chan, "A multiphase level set framework for image segmentation using the Mumford and Shah model," Int. J. Comput. Vis. vol. 50, no. 3, pp. 271-293, 2002.

[42] P.-Y. Tsai, H.-C. Chen, H.-H. Huang, C.-H. Chang, P.-S. Fan, C.-I. Huang, Y.-C. Cheng, F.-M. Chang, and Y.-N. Sun, "A new automatic algorithm to extract craniofacial measurements from fetal three-dimensional volumes," Ultrasound Obst. Gynecol., vol. 39, pp. 642-647, 2012.

[43] R. Xu, O. Michailovich, and M. Salama, "Information tracking approach to segmentation of ultrasound imagery of the prostate," IEEE Trans. Ultrason., Ferroelectr., Freq. Control, vol. 57, no. 8, pp. 1748-1761, Aug. 2010.

[44] W. Lee, R. Deter, B. McNie, M. Powell, M. Balasubramaniam, L. Goncalves, J. Espinoza, and R. Romero, "Quantitative and morphological assessment of early gestational sacs using three-dimensional ultrasonography," Ultrasound Obst. Gynecol., vol. 28, no. 3, pp. 255-260, 2006

[45] J. Anquez, T. Boubekeur, L. Bibin, E. D. Angelini, and I. Bloch, "Uterofetal unit and pregnant woman modeling using a computer graphics approach for dosimetry studies," in Proc. Med. Image Comput. Comput.-Assisted Intervention, London, U.K., Sep. 2009, vol. LNCS5761, pp. 1025-1032.

[46] L. Bibin, J. Anquez, J. P. De La Plata Alcalde, T. Boubekeur, E. D. Angelini, and I. Bloch, "Whole body pregnant woman modeling by digital geometry processing with detailed utero-fetal unit based on medical images," IEEE Trans. Biomed. Eng., vol. 57, no. 10, pp. 2346-2358, Oct. 2010.

[47] L. Vese and T. Chan, "A multiphase level set framework for image segmentation using the Mumford and Shah model," Int. J. Comput. Vis., vol. 50, pp. 271-293, 2002.

Authors' photographs and biographies not available at the time of publication. 